\title{
Broadband Passive InP Membrane Regenerator for Silicon-based Optical Interconnect Applications
}

\author{
M. Tassaert ${ }^{1}$, H.J.S. Dorren ${ }^{2}$, G. Roelkens ${ }^{1}$, and O. Raz ${ }^{2}$ \\ 1. INTEC, Ghent University-imec, Sint-Pietersnieuwstraat 41, 9000 Ghent, Belgium \\ 2. Eindhoven University of Technology, Den Dolech 2, 5600MB, Eindhoven, The Netherlands
}

\begin{abstract}
Improved passive signal regeneration performance based on bonded InP membrane waveguides is demonstrated. A tripling of the ER and receiver sensitivity enhancement of $>3.6 \mathrm{~dB}$ is achieved over the entire C-band at a bitrate of $2.5 \mathrm{~Gb} / \mathrm{s}$.

OCIS codes: (130.3120) Integrated optics devices; (200.6015) Signal regeneration
\end{abstract}

\section{Introduction}

The current trend in high-performance computing is towards parallelism on all levels. As the number of devices working together increases, the amount of data that needs to be sent on and off chip increases as well. Electronics however, are fundamentally limited in the achievable bandwidth-distance product and therefore alternative solutions need to be found. With the advances in photonic integration technology, an integrated optical solution has become feasible. However, for such an integrated solution to be viable, the fabrication should be cost effective and as far as possible CMOS compatible. In this context, silicon photonics has seen increasing interest from industry and academia [1-2]. Although silicon is an excellent material to achieve passive optical functions, the realization of active functions is more challenging due to silicon's indirect band gap. Using heterogeneous integration of III-V on silicon, light sources and detectors have already been successfully demonstrated [1]. Another critical component for the realization of on-chip optical interconnects is the optical modulator. Using the plasma dispersion effect in silicon, several silicon devices have been achieved based on resonant cavities. Although these devices operate at high speed and have low power consumption [2], the intrinsically narrow optical bandwidth makes these devices vulnerable to temperature and fabrication variations. To allow broadband operation, solutions based on MachZehnder interferometers have been proposed. The drawback of these however, is that the power consumption is higher: typical devices show a $V_{\pi} L_{\pi}$ product of 1 Volt $x \mathrm{~cm}$ [3], which means that devices need to be either very long or operate at high voltages to achieve a good extinction ratio (ER) in the modulated optical signal. Furthermore, the free carrier absorption effect causes considerable insertion losses. To make optical interconnects competitive with their electrical counterpart, the power consumption should be brought down considerably [4], which corresponds to a reduction of the operating voltage and device length of the modulator. This implies however that the achieved ER in the modulated signal will be low, leading to a high receiver power penalty. Therefore, it would be beneficial to include a component that can increase the ER of a signal and that requires a low operating power to do so.

Previously we have demonstrated the use of the Membrane InP Switch (MIPS) as a passive low-power regenerator that can be integrated on the silicon-on-insulator platform [5]. Based on recent improvement in device design and fabrication we report in this paper a faster, more power efficient and broadband device. Operation over the entire C-band with a minimal receiver sensitivity enhancement of $3.6 \mathrm{~dB}$ (and up to $4.5 \mathrm{~dB}$ ) is achieved at a bitrate of $2.5 \mathrm{~Gb} / \mathrm{s}$ in a device of only $100 \mu \mathrm{m}$ long.

\section{Device layout}

The device layout of the MIPS is schematically shown in figure 1(a). The basic layout is very similar to the previously introduced device [5], consisting of a $100 \mathrm{~nm}$ thick InP membrane rib waveguide coupled to a $220 \mathrm{~nm}$ thick underlying silicon circuit using two $18 \mu \mathrm{m}$ long inverted taper couplers to ensure adiabatic coupling of the fundamental TE-mode. However, compared to the previous device the quantum well (QW) stack was slightly changed: instead of $8 \mathrm{~nm}$ InGaAs QWs, $10 \mathrm{~nm}$ InGaAs QWs were used. This has resulted in a shift of the band edge from $1580 \mathrm{~nm}$ to $1620 \mathrm{~nm}$, which yields a higher, more uniform light absorption across the C-band. Next to this, the width of the membrane rib waveguide is brought down from $2 \mu \mathrm{m}$ to $1 \mu \mathrm{m}$. This leads to a reduction of the total QW volume with $50 \%$, allowing for a $50 \%$ reduction of required energy to bleach the device while the confinement of light inside the QWs and thus the functionality of the device remains nearly the same. Furthermore, this brings the 


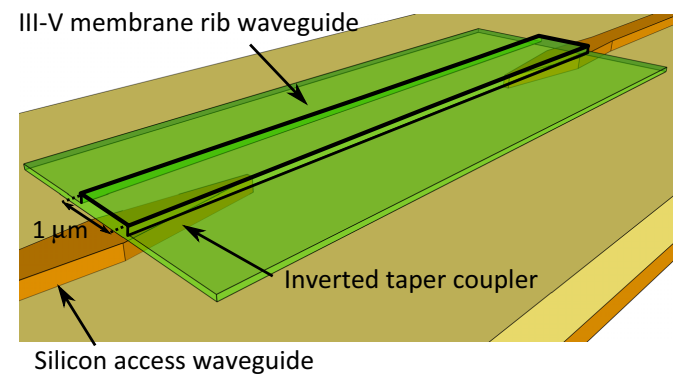

(a)

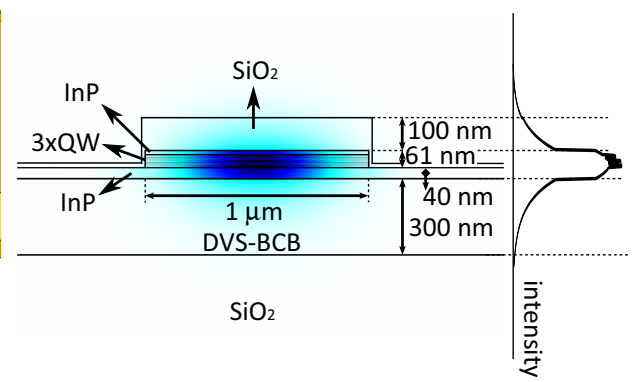

(b)

Fig. 1. (a) Schematic view of the device. (b) Calculated mode profile in the device and cross section of the intensity in the center of the device.

etched QW interfaces closer towards the mode center, increasing the amount of surface recombination of the generated carriers and therefore reducing the carrier lifetime. Since similar to the previous device the speed was fundamentally limited by the carrier lifetime, this led to a faster device as will be shown below. The mode profile in the membrane rib waveguide is shown in figure 1(b). Due to the use of a different QW stack and waveguide width, the confinement of light in the QWs is as high as $22 \%$, an improvement of $5 \%$ over the previous design. The higher confinement in combination with the higher absorption in the C-band of the QW stack allows for a reduction of the total device length from $150 \mu \mathrm{m}$ to $100 \mu \mathrm{m}$.

The high confinement makes the MIPS ideal for use as a saturable absorber. This is the case because the differential modal absorption $\partial \alpha / \partial N$ of the device is proportional to the confinement inside the QWs. Therefore, the device absorption will rapidly change with changing carrier concentration $(N)$. In turn, these carriers are created by the absorption of the signal beam itself, which is again proportional to the confinement in the QWs.

\section{Device characteristics}

In figure 2(a) the $\mathrm{CW}$ device transmission curves are shown for different wavelengths across the C-band. In these transmission curves, three domains can be identified. For low signal powers, there is a quasi-linear relationship between input and output power, as the amount of generated carriers is insufficient to significantly change the high device absorption $\left(\alpha\left(P_{i n}\right) \approx \alpha_{0}\right)$. Also for high signal powers the transmission curves show this proportional relationship, as the amount of generated carriers is sufficient to bleach the absorption completely $\left(\alpha\left(P_{i n}\right) \approx 0\right)$. For moderate powers however, there is a transition between these two regions, which leads to a region where the slope of the transmission curve $\left(\partial P_{\text {out }} / \partial P_{i n}\right)$ is higher than one and values of more than a factor of four are achieved. In this transition region, signal regeneration is possible.

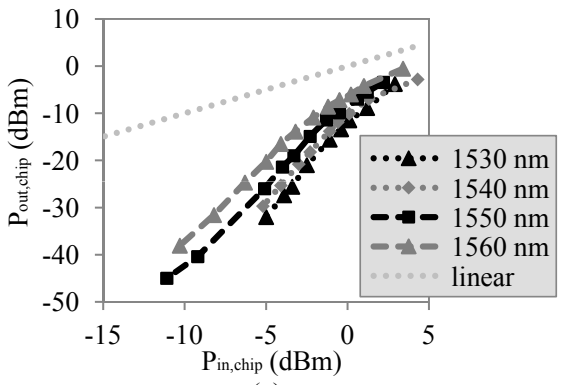

(a)

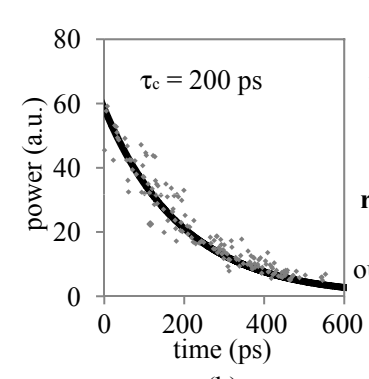

(b)

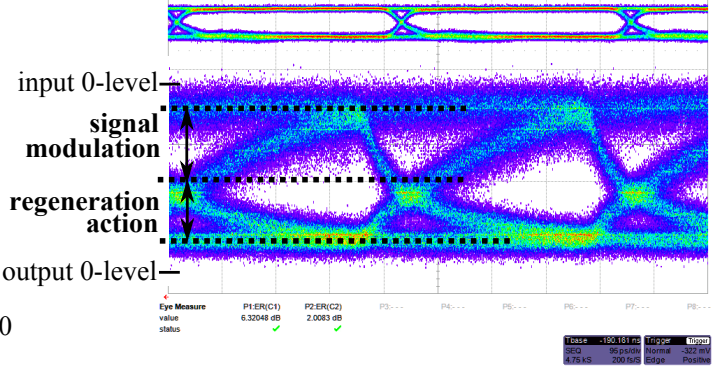

(c)

Fig. 2. (a) CW transmission through the device (excluding coupling losses) (b) Trace showing the carrier lifetime (c) Eye diagram: the top eye is the input signal, the bottom eye is the regenerated output signal. The device draws the signal zero-level closer to the real zero-level.

To assess the device speed, a femtosecond pulse train with high peak power and center wavelength at $1550 \mathrm{~nm}$ was sent together with a weak CW probe bream at a wavelength of $1590 \mathrm{~nm}$ through the device. When such a femtosecond pulse is transmitted through the device, a large number of carriers are created quasi-instantaneously. This causes an immediate bleaching of the absorption of the probe signal, which can be monitored on an optical oscilloscope. By fitting the exponential roll-off of the probe transmission due to the recombination of the created carriers, the carrier lifetime was determined to be 200 ps (see figure 2(b)), an improvement of a factor of three compared to the previous design. Three possible causes can be identified for this lower carrier lifetime. First of all, the used QW stack has changed. Secondly, the waveguide was etched more aggressively during dry etching to introduce more defects at the etched QW interfaces. Finally, the narrower device width also has a significant 
contribution. To determine the effect of the width, the carrier lifetime was also measured for a $1.5 \mu \mathrm{m}$ broad device

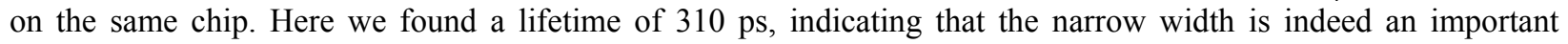
contribution.

\section{Regenerator performance}

To test the regenerator performance, a bit error rate (BER) measurement was performed at a speed of $2.5 \mathrm{~Gb} / \mathrm{s}$. In this measurement a pseudorandom bit sequence of $2^{31}-1$ bits was imprinted on a $\mathrm{CW}$ signal carrier using non-return to zero on-off keying (NRZ-OOK) with an ER of only $2 \mathrm{~dB}$.
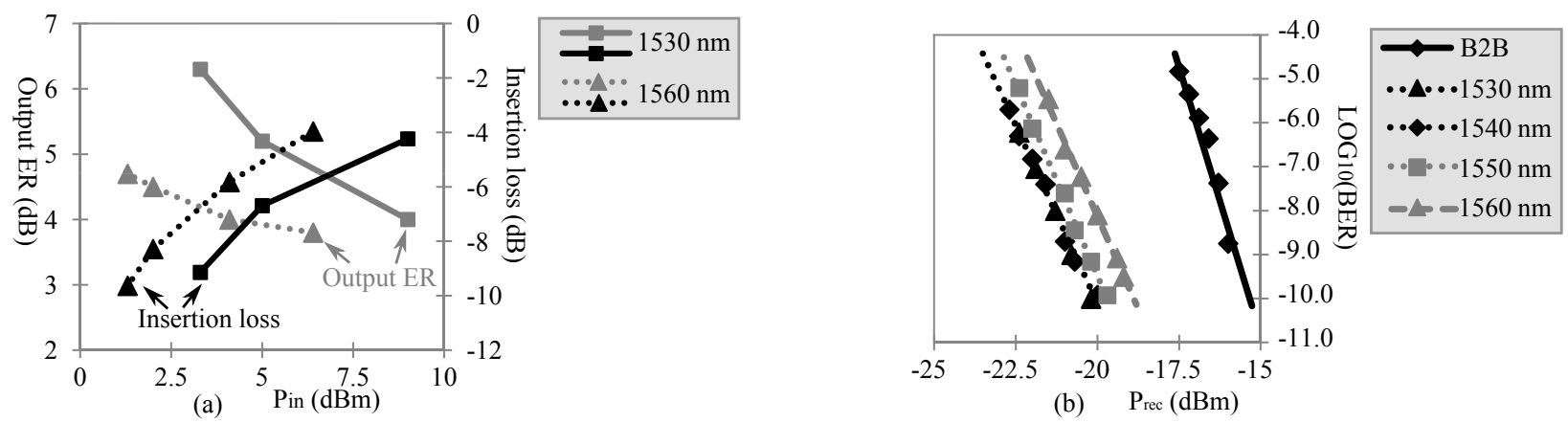

Fig. 3. (a) Output ER and insertion loss as a function of signal input power. (b) BER measurement as a function of received power.

As can be derived from the $\mathrm{CW}$ device characteristics, the regeneration performance will depend on the input power of the signal. Therefore, in a first step the optimal working point for regeneration needs to be determined as a function of wavelength. To do this, the signal is sent through the chip at different input powers and the resulting ER of the output signal is recorded by measuring the eye pattern. Furthermore, by comparing the measured output power to a reference measurement the insertion loss of the device is determined. In figure 3(a) the ER and insertion loss are shown for a signal wavelength of $1530 \mathrm{~nm}$ and $1560 \mathrm{~nm}$, and one corresponding eye diagram is shown in figure 2(c). Unfortunately, there seems to be a trade-off between the regeneration performance of the device and the insertion loss. To avoid high insertion losses, a high power should be applied to bleach the device absorption. However on the other hand, to have good regeneration action, the input power should be lower, such that the working point lies in the region with high slope $\left(\partial P_{\text {out }} \partial P_{\text {in }}>1\right)$. Next to this, it can be seen that the device performs well over the entire C-band.

The results from the BER measurements are shown in figure 3(b). As a working point, we have chosen for an optimal regeneration with an insertion loss of $9 \mathrm{~dB}$ for all wavelengths. Compared to the back-to-back (B2B) measurement, this yields a receiver sensitivity improvement of over $3.6 \mathrm{~dB}$ over the entire C-band up to $4.5 \mathrm{~dB}$ for the signal at a wavelength of $1530 \mathrm{~nm}$ and $1540 \mathrm{~nm}$.

\section{Conclusion}

In this paper, we have shown a large improvement in the regenerator performance of the MIPS. Due to a number of design improvements, the current device is faster, more power efficient and broadband, with a demonstrated $3.6 \mathrm{~dB}$ receiver sensitivity enhancement over the entire C-band at $2.5 \mathrm{~Gb} / \mathrm{s}$. In future work, the device will be further sped up by using ion implantation to decrease the carrier lifetime below $100 \mathrm{ps}$. This should then allow for $10 \mathrm{~Gb} / \mathrm{s}$ operation.

\section{References}

[1] G. Roelkens, L. Liu, D. Liang, R. Jones, A. Fang, B. Koch, and J. Bowers, "III-V/silicon photonics for on-chip and inter-chip optical interconnects," Laser Photon. Rev. 4, 751-779 (2010).

[2] G. Reed, G. Mashanovich, F. Gardes, and D. Thomson, "Silicon optical modulators," Nat. Photonics 4, 518-526 (2010).

[3] L. Liao, D. Samara-Rubio, M. Morse, A. Liu, D. Hodge, D. Rubinm, U. D. Keil, and T. Franck, "High speed silicon Mach-Zehnder modulator," Opt. Express 13, 3129-3135 (2005).

[4] D. Miller, "Device requirements for optical interconnects to silicon chips," Proc. IEEE 97, 1166-1185 (2009).

[5] M. Tassaert, H. Dorren, G. Roelkens, and O. Raz, "Passive InP regenerator integrated on SOI for the support of broadband silicon modulators,” Opt. Express 20, 11383-11388 (2012). 